\title{
Maternal Rubella Susceptibility and Autism in Offspring: Case-Control Study of Maternal Non-Immunity and Low Immunity
}

\author{
Jill Hutton ${ }^{1}$, Paul J. Rowan ${ }^{2}$, Anthony Greisinger ${ }^{3}$ and Melanie Mouzoon ${ }^{4}$ \\ ${ }^{1}$ The Woman's Hospital of Texas, USA \\ ${ }^{2}$ The University of Texas-Houston School of Public Health, USA \\ ${ }^{3} \mathrm{Kel}$ sey Research Foundation, USA \\ ${ }^{4}$ Kelsey-Seybold Clinic, USA
}

"Corresponding author: Paul J. Rowan, The University of Texas-Houston School of Public Health, USA, Tel: 7135009183, E-mail: Paul.J.Rowan@uth.tmc.edu

Received date: Apr 30, 2014, Accepted date: Aug 17, 2014, published date: Aug 24, 2014

Copyright: (c) 2014 Hutton J, et al. This is an open-access article distributed under the terms of the Creative Commons Attribution License, which permits unrestricted use, distribution, and reproduction in any medium, provided the original author and source are credited.

\begin{abstract}
Objective: Autism may arise from any of several categories of harm, including genetic, environmental, perinatal, and drug exposures. Rubella has been examined as one of the viral causes of autism. We designed a study to determine whether antenatal susceptibility to rubella, shown by low or no immunity when tested in the antepartum, was associated with child autism spectrum disorders.

Study Design: Children ages 2.5 years to 7.5 years with autism spectrum disorder were identified, and matched on age and sex with children having no such diagnosis. To identify mothers who were rubella-susceptible, we noted those with pregnancy rubella IgG values under $10 \mathrm{IU} / \mathrm{mL}$; since low-immunity might also pose a risk, we analyzed those with $\mathrm{lgG}$ values under $20 \mathrm{IU} / \mathrm{mL}$. Exclusion criteria included preterm delivery, child brain injury or genetic disorder, and maternal use of anti-epileptic or illicit drugs.

Results: For the years 2007 to 2011 , we identified 56 children with autism meeting study criteria, and identified suitable children as matched controls. Of the 56 autism-case mothers, one had a rubella lgG value under $10 \mathrm{IU} / \mathrm{mL}$, while 6 of the control mothers had an IgG value under $10 \mathrm{lU} / \mathrm{mL}$. For the low-immunity group, 19 of the autism-case mothers had an IgG value under $20 \mathrm{IU} / \mathrm{mL}$, while 18 of the control mothers had an $\mathrm{IgG}$ value under $20 \mathrm{IU} / \mathrm{mL}$. These associations were tested with McNemar's exact (binomial) test. There was no statistical relationship between the presence of an autism diagnosis and mother's rubella susceptibility at the $<10 \mathrm{IU} / \mathrm{mL}$ level $(p=0.13)$, and at the $<20$ IU/mL level $(p=0.85)$

Conclusions: We failed to find evidence supporting the concept that antenatal rubella susceptibility was associated with child autism. Results are not conclusive since this exploratory study was under-powered. We believe that the hypothesis warrants more investigation, including studies with greater power and complementary approaches.
\end{abstract}

\section{Introduction}

The prevalence of autism spectrum disorders has been estimated at one in 68 children [1]. The causes of autism are unclear; autism may arise from any of several categories of harm, including genetic, environmental, perinatal, and drug exposures [2]. Autism is fairly heterogeneous in its presentation, including in symptoms and in severity. This is likely due to the fact that multiple genes are necessary for autism to emerge, in combination with a host of possible environmentally-introduced stressors or insults [3].

One avenue of exposure is viral disease. Dietert et al. [4] note that both the first and second trimesters may be critical windows of vulnerability for autism in the presence of maternal viral infection. For example, data have implicated herpes simplex virus and cytomegalovirus [3], among others.

Rubella has also been examined as a viral cause of autism. While Congenital Rubella Syndrome (CRS) is the most commonly recognized sequela of in utero rubella exposure, other sequelae have also been suspected. The 1964 rubella epidemic gave rise to the reporting of many observations, often case studies or studies of small numbers of patients. This wave of investigation suggested that autism, and other neurological problems, may be seen in the children of women who had rubella during pregnancy [5]. That investigation identified autism or autism symptoms in 18 of $243(7.4 \%)$ subjects with congenital rubella syndrome.

These investigations examined outcomes related to recognized cases of rubella during pregnancy. Sub-clinical rubella exposure may be yet another risk factor for autism. The CDC Pink Book [6] notes that "up to $50 \%$ of infections may be subclinical or inapparent."

Women who have received the rubella immunization but who are not immune are likely unaware that they are vulnerable to rubella. The immunization may not have been effective, or immunity may have waned across time. Women may be non-immune, or may be lowimmune, with only a modest level of antibodies to fight against a rubella exposure. Because rubella poses a threat to the developing baby, women of child-bearing age who are not pregnant are encouraged to ensure immunity, and get immunized, especially if they 
decide they want to become pregnant in the near future. Since the immunization is a weakened virus, any woman must avoid getting pregnant for a month following immunization, or must avoid immunization if pregnant. So, rubella-immunity is commonly evaluated when beginning prenatal care in order to immunize susceptible women after delivery.

Recognizing that a portion of the population may be non-immune, we previously conducted a study to ascertain the portion of women without immunity (IgG levels below $10 \mathrm{IU} / \mathrm{mL}$ ) who convert to rubella-exposed during pregnancy [7]. Along with the normal immunity assessment at the beginning of pregnancy, we conducted a repeat test at delivery in a sample of rubella non-immune women in a large, urban maternity hospital. We discovered that $6.38 \%$ of those who were non-immune at the beginning of pregnancy converted to immune-status (IgG $>40 \quad \mathrm{IU} / \mathrm{mL})$, indicating exposure during pregnancy.

So, it is plausible that some portion of autism-spectrum disorder, including less severe presentations, may be due to rubella, often infections that are unrecognized or are sub-clinical. This would be possible in women who are rubella non-immune or low-immune at pregnancy. This possible cause of autism would not be discernible from clinical or administrative data sets: unrecognized or mild infection may not be reported by the patient, and if reported, may not be attributed to rubella.

\section{Objectives}

The objectives of this exploratory study are to determine, with a case-control study, whether mothers of children with autism were more likely than mothers of children without autism to be rubella non-immune, or low-immune, during pregnancy. If results were in the hypothesized direction but not statistically significant, a post-hoc power calculation would be conducted to suggest the sample size needed in a future study with greater power.

\section{Research Design/Materials and Methods}

This was a retrospective case-control study of mother-child pairs for children born from 2007 to 2011. The electronic medical record for the Kelsey-Seybold system allowed surveillance beginning in 2007 as a start date for the surveillance period, and selecting 2011 as the end date allowed from 3 to 7 years for autism to be diagnosed and recorded in the electronic medical record. Children with a diagnosis of autism and whose mother's pregnancy care was also documented in the Kelsey-Seybold medical record system were matched, on child age and sex, with mother-child pairs for whom there was no indication of autism. Kelsey-Seybold Clinic is comprised largely of a middle class, insured, non-immigrant population; most any sampling will represent such a population.

Since this healthcare system delivers 2,500 or more babies per year, and assuming an autism-spectrum incidence as great as 1 in 68 , there might be as many as 150 cases of autism in this cohort of children. The data set is unique in allowing maternal immunity and child outcomes to be explored.

Assuming that some children with autism would have not yet been diagnosed, and recognizing that not all mother-child pairs have both obstetric and pediatric care in this healthcare system, we projected that we might identify at least 50 mother-child pairs to test this exploratory hypothesis. The result was expected to indicate whether further investigation of rubella non-immunity, and low-immunity, as a risk factor for autism was reasonable, and to generate an initial effect size estimate for more extensive investigations.

Cases were children with a diagnosis of autism (including autistic spectrum disorder, Asperger Syndrome and pervasive developmental disorder) as documented by ICD codes. Exclusion criteria included: preterm delivery (as higher incidence of brain injury to child); known brain injury of child; known genetic disorder of child (such as Down syndrome or Fragile X), and maternal use of anti-epileptic or illicit drugs. Children with autism-spectrum disorders were matched, on sex and on age (within 6 months) with mother-child pairs with no autismspectrum disorder in the medical record, and otherwise meeting the same inclusion/exclusion criteria.

Maternal pregnancy rubella non-immunity was defined as rubellaspecific IgG less than $10 \mathrm{IU} / \mathrm{mL}$, and rubella low-immunity was defined as rubella-specific IgG less than $20 \mathrm{IU} / \mathrm{mL}$. Rubella nonimmunity is somewhat ill-defined with an arbitrary assignment of rubella specific IgG of less than $10 \mathrm{IU} / \mathrm{mL}$ with the most common technique of enzyme-linked immunosorbant assay (ELISA) [8,9]. Two studies looking at commonly used ELISA tests showed that below 30 $40 \mathrm{IU} / \mathrm{mL}$ is often unreliable $[10,11]$. Furthermore, several studies have emerged showing women with low titers $(<20 \mathrm{IU} / \mathrm{mL}$ by ELISA, and $<1: 32$ by Hemagglutinin Inhibition (HI) correlating to roughly 40 $\mathrm{IU} / \mathrm{mL}$ by ELISA) have contracted rubella and delivered infants with CRS [12-16]. For this reason, $<20 \mathrm{IU} / \mathrm{mL}$ was selected as a lowimmunity value. Any woman with a rubella-specific IgG at or above 20 was considered rubella-immune for the purposes of this study. McNemar's exact (binomial) test [17] was used to determine whether, for these matched pairs, the children with an autism-spectrum disorder diagnosis were more likely to have had a mother with rubella non-immunity (or low-immunity).

\section{Results}

Drawing upon the electronic medical record, 56 children with autistic-spectrum disorders were identified who also had a mother in the medical record system with rubella-specific IgG test data recorded from pregnancy care. This modestly exceeded our anticipated number of 50 . As planned, 56 age-and sex-matched control mother-child pairs were identified.

Of the 56 autism-case mothers, one of 56 had a rubella IgG value under $10 \mathrm{IU} / \mathrm{mL}$, while 6 of the control mothers had an IgG value under $10 \mathrm{IU} / \mathrm{mL}$ (Table 1). Immunity-congruence of these case and control mother-child pairs is shown in Table 1.

For the non-immunity and low-immunity cut-off, 19 of the autismcase mothers had an IgG value under $20 \mathrm{IU} / \mathrm{mL}$, while 18 of the control mothers had an IgG value under $20 \mathrm{IU} / \mathrm{mL}$. Immunitycongruence of these case and control mother-child pairs is shown in Table 2.

Therefore, when the typical $(<10 \mathrm{IU} / \mathrm{mL})$ immunity status level was used, the opposite of the tested hypothesis emerged: non-immunity was more likely in the control-child mothers compared to the autismcase mothers. This observation is compromised by very low frequency counts. With the more inclusive definition of low-immune $(<20 \mathrm{IU} /$ $\mathrm{mL}$ ), the results were in the direction of the hypothesis.

These case-control comparisons were statistically tested with McNemar's exact (binomial) test. For the typical non-immune level of $<10 \mathrm{IU} / \mathrm{mL}$, the two-tailed exact significance was 0.13 : not statistically 
significant, but trending toward non-immunity being protective for autism. Since that is quite illogical, this analysis is more than likely a spurious observation arising from a small sample. With the lowimmunity level of $<20 \mathrm{IU} / \mathrm{mL}$, McNemar's exact (binomial) test resulted in a two-tailed probability of 0.85 ; although this result was in the direction of the hypothesis, this result is not statistically significant.

\begin{tabular}{|l|l|l|l|l|}
\hline & & \multicolumn{2}{|l|}{ Non-Autistic Controls: } & \\
\hline & & Immune Mother & $\begin{array}{l}\text { Non-immune } \\
\text { mother }\end{array}$ & Total \\
\hline $\begin{array}{l}\text { Autism } \\
\text { Cases: }\end{array}$ & $\begin{array}{l}\text { Immune } \\
\text { Mother }\end{array}$ & 49 & 6 & 55 \\
\cline { 2 - 5 } & $\begin{array}{l}\text { Non-immune } \\
\text { mother }\end{array}$ & 1 & 0 & 1 \\
\hline & Total & 50 & 6 & 56 \\
\hline
\end{tabular}

Table 1: Pair Congruence, Child Autism Diagnoses and Presence of Rubella Non-Immunity, 56 Matched Mother-Child Pairs.

\begin{tabular}{|l|l|l|l|l|}
\hline & & \multicolumn{2}{|l|}{ Non-Autistic Controls: } & \\
\hline & & Immune Mother & $\begin{array}{l}\text { Low-immune } \\
\text { mother }\end{array}$ & Total \\
\hline Autism Cases: & $\begin{array}{l}\text { Immune } \\
\text { Mother }\end{array}$ & 23 & 14 & 37 \\
\cline { 2 - 5 } & $\begin{array}{l}\text { Low- } \\
\text { immune } \\
\text { mother }\end{array}$ & 15 & 4 & 19 \\
\hline & Total & 38 & 18 & 56 \\
\hline
\end{tabular}

Table 2: Pair Congruence, Child Autism Diagnoses and Presence of Rubella Non-Immunity or Low-Immunity, 56 Matched Mother-Child Pairs.

To assess how underpowered the finding was with the level of $<20$ $\mathrm{IU} / \mathrm{mL}$, a post-hoc case-control power analysis was calculated with the given data. This might be used by others considering a test of this hypothesis with greater power.

Assuming that the low-immunity value of $<20 \mathrm{IU} / \mathrm{mL}$ has fair validity as an indicator of risk for rubella exposure, and assuming the obtained ratios of autistic spectrum disorders are somewhat representative of the hypothetical relationship explored in this study, we calculated a sample size analysis to determine what sample size would be needed, with this pattern of findings, to be statistically significant. Following the case-control sample-size formula from Fleiss [18], and assuming a 1:1 case/control ratio, we estimated that 570 cases and 570 controls would be needed to have the obtained difference in maternal low-immunity be statistically significant.

\section{Discussion}

There have been a few explorations, with diverse samples and methodology, of rubella as a risk factor for autism-spectrum disorders. For all of these, in utero exposure was noted by mothers with recognized rubella during pregnancy. None have investigated "subclinical or inapparent" exposure, although this type of exposure could be harmful. Both rubella vulnerability and subclinical rubella exposure may be increasing. In this study, we evaluated whether rubella-vulnerability was a risk factor for autism spectrum disorders.

In our recent study [6], we found that $6.38 \%$ of women tested as non-immune at the beginning of pregnancy care converted to rubellaexposed when re-tested at delivery. This re-testing at delivery is not a normal part of obstetric care; if subclinical rubella exposure can cause autistic-spectrum disorders, cases of subclinical rubella exposure in utero are being misclassified as non-exposed. This may be a misclassification bias in existing studies; not only would it weaken the ability to detect a true signal, it would bias results toward the opposite finding, since the cases of autism with no recognized maternal rubella would count as contrary cases. This is problematic since it is widely believed that the cause of autistic spectrum disorders are modest exposures, and are multi-factorial, and are likely cumulative.

In our large outpatient healthcare delivery setting, we were able to draw upon relevant clinical data from several recent years of electronic medical record experience, covering thousands of mother-child pairs, to form a cohort of more than 50 young children with documented autistic-spectrum disorders for whom maternal antepartum rubella immunity data were known. We conducted this exploratory casecontrol test of whether rubella non-immunity, and rubella lowimmunity, might be predictors of autism in order to evaluate evidence and to share the concept, and a post-hoc power calculation, with others.

We found few cases, overall, of autism, as would be expected. We also found few cases of non-immunity (and low immunity), as would be expected; both are infrequent events. This case-control study failed to detect a statistically significant indication that maternal rubella nonimmunity is associated with autism in the young offspring of these mothers.

Along with low power, autism case ascertainment may be a weakness. The ages of the children involved in this study ranged from 2.5 years to 7.5 years, with fewer cases encountered at younger ages. Not all cases of autism may have been detected, and some detected cases may not have been noted in the electronic medical record. This would be likely if a family has recently had no reason for a pediatric visit while addressing a psychiatric problem. Also, "soft" neurological symptoms caused by subclinical rubella exposure might not have crossed the diagnostic threshold well enough to lead to pediatric chart documentation. While the controls were age-matched, which should control for the effect of increasing detection with greater child age, this under-detection bias may have been involved. Future studies could limit this bias if they are able to focus on mother-child pairs in which the children are older, as long as immune-status of the mother is available.

Although the finding was negative, we believe that the hypothesis warrants more investigation, including with greater mother-child sample sizes. The idea is plausible, and our result was not wellpowered. Such subsequent studies would indicate whether our finding, an absolute finding that went in the desired direction but was not statistically significant $(\mathrm{p}=0.85)$ was a weak signal or was merely noise.

The post-hoc power analysis indicated that a case-control study with a 1:1 match would need 570 cases. If the recently noted incidence of autistic spectrum disorders is one case in 68 children, then a general population sample of 38,760 would be needed to yield 570 cases. Overall, while rubella vulnerability may lead to sub-clinical exposure, and that lead to autism-spectrum disorders or symptoms, epidemiological detection of this phenomenon is challenging. If a 
Citation: Hutton J, Rowan PJ, Greisinger A, Mouzoon M (2014) Maternal Rubella Susceptibility and Autism in Offspring: Case-Control Study of

relationship does exist, better measures and symptom detection, as well as complementary research designs, would greatly improve the feasibility of detecting this plausible relationship. Future studies could utilize more matching criteria, such as socioeconomic status or race/ ethnicity, in order to minimize statistical noise from other contributors to autism. In the Kelsey-Seybold Clinic system, it has only recently become routine to gather race/ethnicity, and socioeconomic status is not gathered.

Congenital rubella syndrome has been noted in the children of mothers with previously established immunity [12-16]. This may be happening because immunity has waned in the mother, the immune response was not strong enough, or their antibodies, though adequately measured, do not neutralize rubella [9]. An interesting finding of the study was that about $34 \%$ of all the mothers in the study had low-immunity (IgG<20 IU.ml); this is of uncertain significance. Kelsey-Seybold, in Houston, Texas, is comprised largely of a middle class, insured, non-immigrant population; thus the study sample represents this sector of the population. The low-immunity to rubella affecting $34 \%$ of all mothers in this study is an opportunity for infection. If the incidence of rubella exposure is increasing, it may become necessary for women of child-bearing age to have a follow-up test, after immunization, to assure immunity. Further, it would be wise to re-immunize women found to have low-immunity.

Using medical record-based ICD codes for inclusions and exclusions could be a source or error, since these records are not always complete or accurate, especially for psychiatric conditions, such as illicit drug dependence. Stigma, and the administrative gulf between behavioral healthcare and medical healthcare, can interfere with accuracy of substance dependence documentation in the medical record.

This study reports no statistically significant correlation between rubella-susceptibility of mothers in pregnancy and subsequent autism in their children, but it is underpowered. Further studies on the topic of rubella in pregnancy are needed to reveal its impact on autism and other developmental delays in children.

\section{References}

1. Autism and Developmental Disabilities Monitoring Network Surveillance Year 2010 Principal Investigators, Centers for Disease Control and Prevention (2014) Prevalence of Autism Spectrum Disorder Among Children Aged 8 Years-Autism and Developmental Disabilities Monitoring Network, 11 Sites, United States, 2010. Morbidity and Mortality Weekly Report Surveillance Summaries 63: 6 .
2. Chaste P, Leboyer M (2012) Autism risk factors: genes, environment, and gene-environment interactions. Dialogues Clin Neurosci 14: 281-292.

3. Libbey JE, Sweeten TL, McMahon WM, Fujinami RS (2005) Autistic disorder and viral infections. J Neurovirol 11: 1-10.

4. Dietert RR, Dietert JM, Dewitt JC (2011) Environmental risk factors for autism. Emerg Health Threats J 4: 7111.

5. Chess S (1971) Autism in children with congenital rubella. J Autism Child Schizophr 1: 33-47.

6. Centers for Disease Control and Prevention (2009) Rubella. Epidemiology and Prevention of Vaccine-Preventable Diseases (The Pink Book). 11th ed. Washington, DC: Public Health Foundation, 257-271.

7. Hutton J, Rowan P, Greisinger A, Mouzoon M (2014) Rubella monitoring in pregnancy as a means for evaluating a possible reemergence of rubella. Am J Obstet Gynecol .

8. Skendzel LP (1996) Rubella immunity. Defining the level of protective antibody. Am J Clin Pathol 106: 170-174.

9. Robinson JL, Lee BE, Preiksaitis JK, Plitt S, Tipples GA (2006) Prevention of congenital rubella syndrome--what makes sense in 2006? Epidemiol Rev 28: 81-87.

10. Dimech W, Panagiotopoulos L, Francis B, Laven N, Marler J, et al. (2008) Evaluation of eight anti-rubella virus immunoglobulin $\mathrm{G}$ immunoassays that report results in international units per milliliter. Journal of Clinical Microbiology 46: 1955-1960.

11. Dimech W, Arachchi N, Cai J, Sahin T, Wilson K (2013) Investigation into low-level anti-rubella virus IgG results reported by commercial immunoassays. Clin Vaccine Immunol 20: 255-261.

12. Ushida M, Katow S, Furukawa S (2003) Congenital rubella syndrome due to infection after maternal antibody conversion with vaccine. Jpn J Infect Dis 56: 68-69.

13. Aboudy Y, Barnea B, Yosef L, Frank T, Mendelson E (2000) Clinical rubella reinfection during pregnancy in a previously vaccinated woman. J Infect 41: 187-189.

14. Best JM, Banatvala JE, Morgan-Capner P, Miller E (1989) Fetal infection after maternal reinfection with rubella: criteria for defining reinfection. BMJ 299: 773-775.

15. Banerji A, Ford-Jones EL, Kelly E, Robinson JL (2005) Congenital rubella syndrome despite maternal antibodies. CMAJ 172: 1678-1679.

16. Miron D, On A (1992) Congenital rubella syndrome after maternal immunization. Harefuah 122: 291-293.

17. Breslow, NE, Day, NE (1980) Statistical Methods in Cancer Research. Lyon, France.

18. Fleiss J (1981) Statistical methods for rates and proportions. John Wiley and Sons, New York, USA. 